\title{
Protée
}

\section{Le silence sourd}

\section{Michel Poizat}

Volume 28, numéro 2, 2000

\section{Le silence}

URI : https://id.erudit.org/iderudit/030589ar

DOI : https://doi.org/10.7202/030589ar

Aller au sommaire du numéro

\section{Éditeur(s)}

Département des arts et lettres - Université du Québec à Chicoutimi

\section{ISSN}

0300-3523 (imprimé)

1708-2307 (numérique)

Découvrir la revue

\section{Citer cet article}

Poizat, M. (2000). Le silence sourd. Protée, 28(2), 7-15.

https://doi.org/10.7202/030589ar

\section{Résumé de l'article}

Le silence est systématiquement associé à la situation du sourd (sourd de naissance, dit "muet ») pour de bonnes et de mauvaises raisons. Pour de mauvaises raisons car la réalité de la situation du sourd est loin de pouvoir être qualifiée de « silencieuse ». Mais aussi pour de bonnes raisons: le sourd fait en quelque sorte résonner chez l'entendant un certain silence suscitant particulièrement le rapport du corps au signifiant, faisant valoir le silence comme présence pulsionnelle du corps dans une énonciation langagière et donc comme présentification pulsionnelle de la voix. Paradoxalement, c'est aussi le rapport de la musique au silence qui s'en trouve éclairé.
Ce document est protégé par la loi sur le droit d'auteur. L'utilisation des services d'Érudit (y compris la reproduction) est assujettie à sa politique d'utilisation que vous pouvez consulter en ligne.

https://apropos.erudit.org/fr/usagers/politique-dutilisation/ 


\title{
LE SILEN CE SO U RD
}

\author{
MICHEL POIZAT
}

Le sourd ${ }^{1}$ est systématiquement renvoyé au silence par le monde entendant qui l'entoure: silence qu'il est supposé subir et souffrir, silence qu'il manifeste par sa supposée mutité. En fait, tout comme la nuit de l'aveugle, le silence est une idée qui s'impose à l'entendant confronté au sourd et qui s'impose comme fantasme, car dans la réalité il en va tout autrement. Tous ceux qui sont au contact permanent des sourds peuvent en témoigner: rien n'est moins silencieux que le monde sourd. Comme le souligne cette interprète en langue des signes: «Jamais je n'ai eu l'impression que les sourds étaient dans un monde silencieux, on sait que les sourds c'est particulièrement bruyant " ${ }^{2}$.

Quant à parler de silence pour le sourd, même si c'est un poncif du discours misérabiliste sur cet «infortuné muré dans son silence», rien ne permet non plus de le supposer. Comme le font remarquer à plusieurs reprises les linguistes sourds américains Carol Padden et Tom Humphries ${ }^{3}$ :

Les sourds construisent leur monde à partir de ressources en mouvement, forme et son. La métaphore du silence a un pouvoir explicatif pour les entendants voulant désigner de la sorte ce qui leur paraît être le fait central de l'univers sourd. C'est cependant tout à fait grossier et inadéquat pour rendre compte des connaissances et des actes des sourds.

Nous poursuivons la citation dans sa version originale pour lui garder tout son relief... sonore: "The lives of Deaf people are far from silent but very loudly click, buzy, swish, pop, roar, and whir!».

Reportons-nous également à ce que dit le sociologue B. Mottez concernant cette évocation répétitive du silence, à propos des sourds:

Nous préférons le terme de visuels à celui de silencieux (si usité soit-il) pour désigner les sourds. Nous préférons celui de monde visuel à celui de monde de silence pour désigner le monde des sourds ou leur façon d'être au monde. Ceci parce que le sens du mot silence prête à malentendus tant sont

différentes les expériences qu'on peut en avoir selon qu'on est sourd, malentendant, devenu sourd ou entendant [...]

Penser d'autre part que le sourd de naissance vit dans le silence - à la façon dont nous l'entendons si l'on peut dire - est aussi absurde que l'idée que se font beaucoup de voyants que l'aveugle de naissance vit dans le noir... ${ }^{4}$ 
Certes les sourds eux-mêmes ont parfois repris à leur compte cette référence au silence. C'est ainsi qu'au début du XXe siècle le supplément littéraire au journal La Gazette des Sourds-Muets s'intitulait La France silencieuse. Périodiquement se tenait à Paris un "Salon d'Artistes Silencieux». Le développement des associations sportives de sourds justifia la création du journal Le Sportsman silencieux ${ }^{5}$. Il n'en reste pas moins que, comme nous l'avons dit, c'est à l'entendant que le silence s'impose dans la rencontre avec le sourd. La traduction française par Les Enfants du silence du titre original de la pièce américaine de Mark Medoff, The Children of a lesser god, en est d'ailleurs un symptôme caractéristique $^{6}$ ! Comme le signe Sarah, l'héroïne de la pièce:

- «Etre sourd n'est pas le contraire d'entendre. C'est un silence rempli de bruit.

- Vraiment?, interroge Jacques, son partenaire entendant, un silence rempli de bruit?

- C'est le cri du printemps brisant le sommeil de l'hiver, répond Sarah."

Pourquoi donc cette insistance de l'entendant à renvoyer le sourd au monde du silence? ? En fait, le sens et l'usage commun du mot silence, en rattachant le mot à l'absence de son ou de bruit, font trop oublier le sens premier et profond du mot tel que l'étymologie le rappelle: le silence, fondamentalement renvoie à un "taire" ou à un "se taire», sens premier du verbe latin silere, racine du mot. Le silence en tant qu'absence de bruit ou de son n'en est que la prise en compte des effets secondaires. Cette racine fondamentale, la langue des signes française (LSF) la retrouve d'ailleurs puisque le signe pour «silence» est tiré du geste familier que l'on fait pour demander à quelqu'un de se taire: l'index posé verticalement en travers des lèvres fermées.

C'est donc en tant qu'il est considéré comme "hors parole», hors parole selon la modalité sonore des entendants, que le sourd se trouve caractérisé par le silence ${ }^{8}$. Et pourtant le sourd parle non pas seulement quand, sous l'apprentissage, pour ne pas dire le dressage, oraliste, il s'exprime oralement selon une voix acoustique, proche de celle de l'entendant, non, il parle aussi lorsque, s'exprimant en langue des signes, il énonce - en silence ${ }^{9}$ - les signifiants gestuels de sa langue. Il pourra du coup signifier le silence d'un signifiant gestuel et il pourra même observer une minute de silence à sa manière, buste fixe, mains immobiles, doigts écartés, immobilisant de la sorte tous les points de l'articulation signifiante gestuelle.

Dans le support corporel de son énonciation gestuelle, le sourd nous présente ainsi la voix qui lui est propre et nous invite à redéfinir ce qu'il en est fondamentalement de la voix: non plus «l'ensemble des sons produits par les vibrations des cordes vocales" ${ }^{10}$, mais la part de corps qu'il faut mettre en jeu pour produire un énoncé signifiant, quelle qu'en soit la modalité sensorielle.

Le sourd est donc au cœur d'une configuration qui articule signifiant, voix, corps et silence. Il nous introduit directement à une réflexion articulant notamment voix et silence qui dépasse totalement la spécificité de la situation du sourd pour s'appliquer en fait à la voix comme telle, aussi bien celle de l'entendant parlant que celle du sourd signant.

\section{VOIX ET SILENCE}

Que la voix fondamentalement soit silence, voilà un énoncé qui mérite quelques éclaircissements tant il semble paradoxal et contraire à l'expérience du sens commun. Il résulte pourtant, phénoménologiquement, logiquement et psychologiquement, des liens qui nouent voix, corps et signifiant.

\section{LA TRANSPARENCE DE LA VOIX}

La parole fait taire la voix, la réduit au silence. Support de l'énonciation discursive, la voix présente en effet la particularité de disparaître littéralement derrière le sens du discours qu'elle énonce. Cette observation peut paraître énigmatique, elle est pourtant d'expérience quotidienne. Quand, par exemple, quelqu'un prend la parole, notre attention est souvent au début captée par les caractéristiques de sa voix, son accent... mais très vite cela disparaît sitôt qu'on fait attention au sens de ce qui est dit, à tel 
point que pour ceux qui, par exemple, sont bilingues, il leur arrive fréquemment d'être incapables de se souvenir en quelle langue telle ou telle chose leur a été dite, alors même que les caractéristiques acoustiques des deux langues sont radicalement différentes et ne peuvent être confondues. Le même phénomène se produit lorsque le support de l'énonciation n'est pas sonore mais gestuel, comme dans une conversation entre sourds en langue des signes. C'est ainsi qu'il arrive fréquemment aux interprètes (français oral/ langue des signes) d'être incapables de dire si tel ou tel échange avec un sourd bilingue (oral/langue des signes) a été tenu dans la langue orale ou en langue des signes ${ }^{11}$.

À l'inverse, si quelque phénomène vient affecter l'énoncé signifiant, du fait par exemple de l'introduction d'une temporalité de l'énonciation étrangère à celle de l'énonciation naturelle, ou bien en perturbant l'articulation par une hauteur mélodique incompatible avec la prononciation de certains phonèmes, alors la voix cesse d'être transparente sous le sens et se réintroduit comme telle. Le chant, la musique, ce que je nomme ici d'un mot, le lyrisme, ne sont jamais que de tels "parasitages» de l'énonciation langagière, ayant pour effet d'opacifier, si l'on peut dire, la voix afin de la rendre perceptible, le plus souvent dans un but esthétique, pour jouir de la voix. C'est en cela qu'ils constituent une "voie royale» pour tenter d'appréhender ce qu'il en est véritablement de la voix. Là encore, le même effet surgit dans la situation de l'énonciation langagière gestuelle. Grâce à une amplitude et à un enchaînement particuliers des signifiants gestuels, le sourd «signant» arrive à produire une sorte de chant gestuel, de chorégraphie, mettant en avant la corporéité du support de son discours, au point de le rendre parfois inintelligible. Aux distinctions saussuriennes signifiant-signifiéréférent, il convient donc d'ajouter en amont, et à un autre niveau, la distinction voix-signifiant.

Il n'y a, en fait, rien de nouveau dans cette observation travaillée depuis bien longtemps par la phénoménologie du langage. C'est ce que Derrida formule à sa manière dans son petit ouvrage La Voix et le phénomène:

[...] Le "corps» phénoménologique du signifiant, écrit-il, semble s'effacer dans le moment même où il est produit. [...] Il se réduit phénoménologiquement lui-même, transforme en pure diaphanéité l'opacité mondaine de son corps. Cet effacement $d u$ corps sensible et de son extériorité est pour la conscience la forme même de la présence immédiate du signifié. 12

C'est ce que saint Augustin formulait lui aussi, il y a plus de 1500 ans, à sa manière, dans son sermon 288 sur la naissance de saint Jean-Baptiste, dans lequel il développe l'extraordinaire métaphore selon laquelle la voix est au Verbe, comme Jean-Baptiste, la voix, est au Christ, le Verbe, s'effaçant derrière lui après l'avoir annoncé ${ }^{13}$.

Cette analyse n'est pas sans rapport avec l'élaboration imaginaire occidentale qui affecte à l'ange l'attribut du silence. En effet, selon les termes de J.-C. Milner dans son livre L'Amour de la langue, «l'Ange est ce qui image le sujet lorsqu'il est réduit à sa seule dimension d'énonciation " 14 , autrement dit à celle de voix, en tant que la voix est support d'une énonciation se distinguant de son contenu. Ce n'est dès lors pas un hasard si la langue des signes a pu être définie comme «la langue des anges». Voici en effet ce que disait l'abbé Fauchet de la langue des signes lors de l'éloge funèbre de l'abbé de l'Épée qu'il prononça en 1790 à la demande de la Commune de Paris:

La précision est incroyable, la rapidité paraît surnaturelle. Nous tâtonnons avec nos paroles, ils volent avec leurs signes. Nos esprits rampent et se trainent dans de longues articulations; les leurs ont des ailes et planent sans ralentissement dans l'immensité de la pensée [...]. Et c'est, en effet, Messieurs, le langage des anges que parlent les disciples de M. de l'Épée. 15

Soixante ans plus tard, en 1852, l'écrivain et pédagogue sourd Ferdinand Berthier reprend cette image:

Quand on en a été témoin (d'un échange en langue des signes), on est forcé de convenir qu'il n'y a que ce langage dans lequel les âmes puissent se réfléchir, s'unir étroitement et se parler comme les anges sans doute se parlent dans le ciel. 16 
Formulation imagée s'il en est de cette transparence silencieuse de la voix pensée comme immédiateté d'une communication universelle échappant à la pesanteur, à la temporalité et à la corporéité de l'énonciation signifiante, autrement dit: fantasme d'une communication hors langage.

Si ces formulations présentent une idéalisation du silence sourd rapporté au divin ou tout au moins à l'angélique, il ne faut pas oublier que bien plus massivement la gestualité silencieuse du sourd fut rapportée au contraire plutôt à une certaine diabolisation. Celle-ci visait également - c'est en tout cas notre hypothèse - la voix gestuelle sourde en tant cette fois que la voix constitue la dimension pulsionnelle d'une énonciation langagière et qu'à ce titre elle se trouve soumise à la régulation que toute société organise à l'égard de toute dynamique pulsionnelle. Or la voix, en tant qu'objet pulsionnel, va là encore nous amener du côté du silence, silence d'une autre nature que celle que nous venons de décrire, un silence pensé cette fois comme asymptote, limite ou même vérité de la voix, telle que la pense l'élaboration lacanienne de la voix définissant celle-ci comme modalité de l'objet $a$.

Lorsqu'il entreprend de prolonger et d'approfondir la théorie freudienne de la notion de pulsion, Lacan est amené à repérer deux objets pulsionnels nouveaux: le regard et la voix. Et plus structurellement, ce qu'il appelle son «démontage de la pulsion» le conduit à souligner la caractéristique principale des objets pulsionnels: tirer leur efficace de ce qu'ils manquent, de leur propriété de vide, de leur nature «a-substantielle» si l'on peut dire. L'objet n'est opératoire qu'en tant qu'il manque, et qu'il manque structurellement et non pas conjoncturellement. À partir donc du moment où la voix est envisagée comme un objet d'une telle nature, c'est en tant que «vide " de la voix, qu'elle se présentifie au sujet de la façon la plus opératoire, autrement dit comme silence. Tout comme le regard vide de l'aveugle propose la forme la plus aboutie de l'objet regard, le silence constitue la modalité la plus authentique de présentification de l'objet voix. De par son mutisme ou son énonciation silencieuse, le sourd se trouve ainsi en position d'incarner littéralement la voix dans sa dimension pulsionnelle. Ce qui permet, entre autres raisons, de mieux comprendre pourquoi il fut à ce point soumis à la pression sociale éducative fondée pour une large part sur un imaginaire le plaçant du côté du sauvage, de l'obscène ou du déchaînement pulsionnel ${ }^{17}$.

Et cela d'autant plus que pour l'entendant confronté au sourd qui ne lui répond pas du fait de sa surdité, se mobilise alors toute la problématique mettant en jeu le silence de l'Autre, ce qu'on pourrait appeler: l'Autre-silence.

\section{L'AUTRE-SILENCE}

C'est toute la dialectique de la parole, telle que l'explicite la psychanalyse dans sa formulation lacanienne qui se trouve ici mise en jeu. Selon les termes mêmes de J. Lacan: "Toute parole appelle réponse. Nous montrerons qu'il n'est pas de parole sans réponse, même si elle ne rencontre que le silence, pourvu qu'elle ait un auditeur» ${ }^{18}$.

Or toute parole suppose en fait un Autre présent à qui l'on s'adresse. Nous disons bien un Autre avec un grand A, qui s'incarne certes dans le "petit autre", l'individu présent à qui l'on s'adresse mais qui ne s'y réduit pas. Le fait qu'on puisse dans certains cas parler tout seul n'infirme en rien la précédente assertion, au contraire: cette situation montre bien qu'il s'agit dans l'acte de parole d'une présence essentielle, non conjoncturelle, indépendante par conséquent de la personne incarnée ou non à qui l'on s'adresse. Cette Altérité foncière, c'est ce que Lacan appelle le «Trésor du signifiant», «lieu du langage». Que le langage émane de l'Autre est en effet une vérité attestée par toute la clinique de «l'appropriation» du langage par le petit d'homme. Or ce grand Autre est absolument supposé entendre et répondre. Même dans le cas où l'on se met à parler tout seul ou pour soi-même, il s'agit bien en fait soit de prendre le ciel à témoin, soit de se prendre soi-même à témoin. Cela présuppose dans tous les cas, d'abord une Altérité foncière, ensuite la capacité prêtée à l'Autre d'entendre et de répondre, ne 
serait-ce que par le silence. Ce dernier devient alors une modalité de réponse n'invalidant en rien le présupposé inconscient selon lequel l'Autre a entendu. Or, que se passe-t-il dans la situation de l'adresse à un sourd (ou de l'adresse du sourd à l'autre, c'est la même chose car le propre de la situation de parole est de se renverser: on ne parle qu'à celui qui peut parler, ou plus exactement qu'à celui qui peut répondre d'une façon ou d'une autre ${ }^{19}$, même si le code social ne lui autorise pas la prise de parole. Ce n'est alors qu'un mutisme conjoncturel, non structurel)? Et lorsque l'autre répond, il attend lui aussi une réponse (réversibilité de la situation) même si cette réponse peut être le silence ou un acte non verbalisé.

Dans le rapport de parole sourd/non-sourd, deux situations peuvent donc se produire. La première est celle du sourd oralisé, parlant donc, qui s'adresse au non-sourd. Ce dernier se croit dès lors dans un type de rapport de parole "classique», présupposant que l'Autre entend et parle. S'il se révèle, dans les difficultés du dialogue et les limites de la lecture labiale du sourd, que brusquement ce dernier cesse d'incarner pour l'entendant cet Autre auquel s'adresse le non-sourd, la parole de celui-ci ne peut alors que défaillir. Une «inquiétante étrangeté» l'envahit, produisant alors ces troubles et affects bien connus de tous ceux qui se sont trouvés un jour dans cette situation: la surdité de l'autre suscite la sidération et la mutité de l'interlocuteur non sourd du fait de l'impossibilité pour celui-ci de soutenir le présupposé inconscient de l'Autre «entendant». Le trouble ne peut alors se dissiper que si la révélation de la surdité est signifiée d'une façon ou d'une autre. Le grand Autre supposé entendre peut alors être restauré dans sa présence "entendante" au simple prix d'une transposition dans le registre du visuel. Il se trouve alors incarné par un "petit autre» sourd, certes, mais «voyant-entendant", par ses yeux, les "gestes-paroles» qui lui sont adressés. Il est important en effet de noter ici qu'au sens strict et étymologique, "entendre" n'implique pas que l'on soit dans le registre de l'ouie. «Entendre» vient en effet du verbe latin intendere qui signifie "tendre vers», d'où «porter son attention vers». Selon Bloch et von Wartburg ${ }^{20}$, ce n'est qu' à partir du XVIIe siècle qu'il tend à se substituer à "ouïr». Le sens étymologique décrit donc très exactement la position occupée par cet Autre, "présent et entendant», dans la structure du rapport de parole. En ce sens le sourd peut donc incarner l'Autre «entendant» aussi bien qu'un non-sourd.

Dans un deuxième type de situation, mettant en scène la rencontre entre un sourd non parlant mais s'adressant d'emblée à l'entendant en langue des signes - deuxième situation dite "gestualiste» -, les choses se passent très différemment: le "petit autre", sourd, du fait de son abord silencieux et gesticulant, s'avère défaillant à incarner d'entrée de jeu, dans l'établissement du rapport de parole, ce grand Autre supposé entendre et répondre ("Que me veut donc cet énergumène - étymologiquement, énergumène signifie "sous influence" - sous-entendu: démoniaque»). Les réactions de fuite et d'évitement - d'esquive - sont massives dans cette situation. Sauf pour ceux pour lesquels cette modalité de l'abord signifie aussitôt la surdité. Dans ce cas, l'interlocuteur peut opérer immédiatement la restauration du rapport de parole "canonique» par la transposition dans le registre du visuel de la structure de ce rapport. C'est donc le rétablissement du statut de cet Autre présent et «entendant", même si c'est selon la modalité visuelle, qui permet à la parole de circuler à nouveau, $y$ compris dans sa modalité sonore pour l'entendant. La structure du rapport de parole s'établit d'emblée et se remanie très vite dans le registre du visuel. Le trouble entraîné par l'inquiétude devant cet Autre étrange en est sinon évité, du moins rapidement dissipé.

Le trouble qui découle de ces dysfonctionnements du rapport de parole n'est pas, soulignons-le, spécifique du rapport de surdité. Il peut surgir dans la relation, par exemple, avec un étranger tout à fait entendant mais ne comprenant pas et ne pas parlant pas notre langue, ou même avec tout entendant. Mais le sourd se trouve quand même tout à fait prédisposé, de par sa surdité, à le susciter. Fondamentalement, ce qui en effet est en jeu ici, c'est le malaise entraîné par 
la non-réponse, par le silence de l'Autre. Mais il faut bien distinguer ici plusieurs silences, de statut et d'effets très différents.

Il y a d'abord le silence de l'absence de réponse, lié à l'incapacité pour l'Autre de répondre. Nous avons vu que lorsque l'on s'adresse à l'Autre, ce dernier est absolument supposé répondre. Son silence, dans ce cas, est la marque de sa défaillance, de son incomplétude. Il avoue son manque de garantie de l'ordre symbolique, il se révèle «barré», castré dans le jargon psychanalytique.

Il y a ensuite un deuxième silence, toujours pour ce qui concerne le rapport de parole. C'est le silence déterminé par le refus de répondre. Quand je m'adresse à l'Autre, c'est que je lui veux quelque chose: je me révèle désirant. Si l'Autre refuse de me répondre, c'est qu'il ne me reconnaît pas, c'est donc que je ne vaux rien: je suis renvoyé à ma propre incomplétude, à ma propre castration. Le malaise m'envahit alors du fait de ce rappel. Ceci explique bien, par exemple, pourquoi le fait pour l'Autre de ne pas répondre à une question ou à une parole qu'on lui adresse (parce qu'il ne l'a pas entendue ou même simplement parce que, cherchant la réponse, il tarde à répondre) a toujours pour effet de susciter un sentiment pouvant se traduire, pour certains sujets particulièrement concernés, par des réactions d'une violence tout à fait disproportionnée, comme si - soudain - leur existence même était en jeu. Ce n'est d'ailleurs pas sans raison que le code des bonnes manières exige qu'on accuse réception d'un message même si l'on n'est pas en mesure d'y répondre. Regardons également ce qui se passe dans le cas du répondeur téléphonique. Ce dernier a, en fait, pour fonction de disjoindre dans le temps l'envoi de l'adresse à l'autre et sa réception: qui, alors, n'a pas éprouvé un certain agacement (pour le moins!) lorsque, après qu'on lui ait laissé un message appelant réponse sur son répondeur, l'Autre correspondant ne se manifeste pas, et semble donc s'y montrer sourd? Ce n'est par un hasard si le Moyen Âge a défini le péché de taciturnitas. Il rapproche celui-ci du péché d'orgueil que l'on commet lorsqu'on se montre hautain devant la parole de l'autre, cette dernière devant s'entendre comme l'expression d'une demande ou d'une souffrance. Avec ses mots, le Moyen Âge a parfaitement compris l'enjeu de désir qui sous-tend le rapport de parole: quand je m'adresse à l'Autre, je m'avoue désirant. Si l'Autre refuse de répondre, c'est qu'il refuse de s'inscrire dans la dialectique du désir, qu'il se pose, ou prétend se poser, comme non désirant, position d'absolu réservée à la toute-puissance divine et dans laquelle aucun être humain parlant ne saurait se placer: «Le péché de taciturnitas est un péché important car il renvoie au choix primordial et fondamental entre la parole et le silence " 21 .

Ce que le Moyen Âge définit ainsi comme péché, c'est la non-reconnaissance de l'Autre en tant que sujet parlant, s'avouant donc de ce fait désirant.

L'expérience du silence, qu'elle soit du premier ou du deuxième type, la brusque confrontation à un Autre que l'on ne peut plus soudain supposer entendre ou répondre, ou que l'on peut même supposer absent, est productrice d'affects ravageurs pour le Sujet puisque, comme nous l'avons vu, c'est de l'Autre, lieu du langage, que nous tirons notre statut de sujet parlant, de "parlêtre». C'est sans doute là l'une des données de la psychose. L'enfant autiste ne peut-il pas ainsi, d'un certain point de vue, être envisagé comme un enfant, certes entendant au sens propre, mais qui fait la sourde oreille et fait de l'Autre aussi bien un être sourd. James Joyce, dont la structure psychotique fut évoquée par Lacan dans son séminaire "Le Sinthomme", disait qu'il n'était superstitieux que d'une chose: des sourds-muets 22 . On comprend pourquoi quand on sait à quel point était fragile - et fondamental - chez lui cet ancrage au langage auquel l'écriture, dans la modalité déchaînée qu'on lui connait, le raccrochait ${ }^{23}$.

L'auteur dramatique allemand Georg Büchner, dans une remarquable intuition de cette problématique, trouve également ces mots extraordinaires qu'il met dans la bouche du poète Lenz dont il raconte le naufrage dans la folie: «Mais [dit Lenz à son ami le pasteur Oberlin], vous n'entendez donc pas cette voix atroce qui hurle tout autour de l'horizon et qu'on appelle d'habitude le silence? "24. 
D'une façon générale d'ailleurs, la thématique développée par Büchner dans ce texte admirable est singulièrement pertinente à notre propos sur la voixobjet et sur le silence de l'Autre, silence de Dieu en l'occurrence, dont Lenz fit un jour l'expérience traumatisante: invoquant Dieu pour sauver la vie d'un enfant qui lui était chère, le silence et l'absence de réponse qui lui furent signifiés par la mort de l'enfant devaient le projeter dans un univers désormais marqué par la sourde et omniprésente menace d'un silence et d'un vide absolu auquel il n'échappa qu'en s'y engouffrant: Lenz se jeta par la fenêtre, dans le vide, dans un ultime effort pour briser ce silence: il s'écrasa dans la cour «avec un bruit si fort qu'il parut impossible à Oberlin que la chute d'un homme en fût la cause ${ }^{25}$.

Il faut d'ailleurs ici se demander si, au-delà de sa préoccupation pédagogique, le jansénisme ne prédisposait (prédestinait!) pas, fondamentalement, l'un des siens - ce fut l'abbé de l'Épée - à se pencher sur la question des sourds-muets, précisément en raison de cette problématique du silence, de la non-réponse de l'Autre. N'est-ce pas, en effet, un Dieu au désir énigmatique et absolu que nous présente la doctrine de la prédestination de Jansénius? Un Dieu sourd-muet, en quelque sorte, réservant le salut aux seules créatures qu'il a choisies et restant sourd et muet, silencieux, envers les autres, quels que soient leurs mérites ou l'ardeur de leurs invocations à son adresse?

C'est ainsi une problématique articulant deux sortes de silence qui se trouve mobilisée par le sourd dans le rapport qu'il entretient avec l'entendant, l'une et l'autre source de malaise, voire d'angoisse, pour l'entendant confronté au sourd: le silence de l'Autre et son absence de garantie de l'ordre symbolique et le silence comme présence pulsionnelle du corps dans une énonciation langagière, c'est-à-dire comme présentification de la voix comme objet pulsionnel.

\section{MusiQue ET SILENCE}

$\mathrm{Or}$ - et c'est la dernière remarque que nous voudrions faire pour conclure, ce point d'articulation ente deux registres du silence se retrouve au cœur même des enjeux fondamentaux de la musique, notamment dans sa modalité sacrée - ce n'est pas le moindre des paradoxes qu'une réflexion sur le silence conduite à partir du silence sourd nous amène à aborder les rapports déjà bien repérés entre la musique et le silence. Et pourtant, n'est-ce pas ce que nous signifie Carson McCullers quand elle appelle le héros sourd-muet de son roman The Heart is a Lonely Hunter, Mr Singer. Remarquons au passage, et ceci prend tout son sens ici, que le destin même de Carson McCullers fut déterminé par un certain rapport à la voix. Carson aurait dû en réalité s'appeler Caruso. Sa mère fut, en effet, tellement subjuguée par la voix de Caruso, entendue alors qu'elle était enceinte, qu'elle décida d'appeler son enfant Caruso. La naissance d'une fille l'amena à adapter Caruso en Carson, prénom qui laisse subsister quand même quelque étrangeté pour une fille aux USA. La petite Carson présenta d'ailleurs des dispositions marquées pour la musique et le piano au point d'envisager d'entrer à la Julliard School. Vacillant entre la machine à écrire et le piano, entre le verbe et la voix, elle opta finalement pour le verbe qui devait la rendre célèbre à vingt-trois ans grâce, précisément, à The Heart is a Lonely Hunter.

Le silence en musique renvoie lui aussi à deux registres différents, l'un qui ressortit à celui du signifiant, et l'autre qui engage plus fondamentalement la question du rapport à une altérité foncière du langage. Le silence est en effet un signifiant musical qui s'écrit sur la partition et se décline sous diverses modalités (soupir, demi-pause, pause). Il participe à ce titre de la scansion de la ligne musicale, tirant la musique du côté du langage en l'identifiant à ce qui, fondamentalement, caractérise tout système de signifiants: la discontinuité introduite dans un continuum sonore pour les signifiants du langage sonore habituel, dans un continuum spatial à deux dimensions pour l'écriture, dans un continuum spatio-gestuel, pourrait-on dire, pour les signifiants gestuels de la langue des signes des sourds, continuum électromagnétique pour le bit informatique...

Mais à l'inverse, toute réintroduction d'une continuité dans ces découpages signifiants a pour 
effet d'abolir ou de dissoudre le signifiant. Or que fait la musique, notamment dans l'art lyrique, sinon réintroduire de la continuité sous diverses modalités dans le discontinu du langage? D'où d'ailleurs l'aporie fondamentale de l'opéra: prima la musica o prima le parole? C'est ce qui produit ce phénomène tout à fait particulier de faire entendre dans l'art lyrique français le e muet, qui est au sens strict inouï. C'est ce qui permet à Vladimir Jankélévitch de décrire la musique en elle-même comme «une manière de silence» ${ }^{26}$. Ce silence-là, dont est porteuse la musique, en contestant, en subvertissant l'ordre de la parole, permet à l'être humain, selon les termes de Jankélévitch, «d'alléger la pesanteur du logos, de desserrer l'hégémonie accablante de la parole», empêchant de la sorte que «l'humain ne s'identifie au parlé» ${ }^{27}$. La musique nous renvoie ainsi à un Autre du langage, silencieux, à la fois apaisant et angoissant. Et la caractérisation sonore la plus parfaite de ce silence même de la musique, c'est en toute logique le son le plus continu possible qui tente de la matérialiser comme nous le donne à entendre le final du Moïse et Aaron, l'opéra resté inachevé de Schönberg ${ }^{28}$. Nombre d'aspects de la problématique que nous avons ici esquissée sont en effet littéralement mis en scène dans le chef-d'œuvre de Schönberg. Moïse, certes n'est pas sourd-muet. De nos jours, il n'aurait cependant pas échappé aux soins d'un orthophoniste!:

"Meine Zunge ist ungelenk

ich kann denken

aber nicht reden " 29

C'est par ces mots qu'il se présente en effet au début de l'œuvre.

"O Wort, du Wort, das mir fehlt!»30: c'est par cette exclamation angoissée, adressée à Dieu, qu'il termine l'œuvre. Un fa dièse tenu très longtemps en point d'orgue diminuendo vient alors prolonger ses paroles.

Parfaite illustration du continuum sonore s'introduisant dans l'empire du mot, cette longue note jouée aux cordes en point d'orgue est peut-être la musicalisation la plus parfaite qui soit de ce silence qui impose sa présence absolue lorsque toute parole défaille. Il est, d'une certaine façon, le pendant du silence originel qui préside à L'Or du Rhin de Richard Wagner. Il dit la vérité que Moïse vient de découvrir sans le savoir: ce manque, ce vide, creusé au cœur même du langage et que les hommes ont tenté de combler par la figure entre autres du Dieu-Verbe, ce vide est silence: Dieu est silence. Plus rien dès lors ne peut être dit, l'œuvre de Schönberg ne peut qu'en rester là, dans l'inachèvement qui caractérise son aboutissement. 


\section{N O TES}

1. Précisons que le terme de sourd utilisé dans cet article désigne toujours le sourd de naissance, celui qu'on appelait autrefois le «sourdmuet", ou à la rigueur le devenu sourd suffisamment précocement pour que l'acquisition du langage ne puisse s'effectuer dans des conditions "normales" (sourd dit "prélingual»).

2. Entretien avec l'auteur. Cf. M. Poizat, La Voix sourde, Paris, Métailié, 1996, p. 62.

3. C. Padden et T. Humphries, Deaf in America. Voices from a Culture, Cambridge (Mass.) et Londres, Harvard University Press, 1988, p. 109, (c'est nous qui traduisons), cf. aussi p.93 et 104 .

4. B. Mottez, La Surdité dans la vie de tous les jours, Paris, Diffusion P.U.F., publication du Centre Technique National d'Études et de Recherche sur les Handicaps et les Inadaptations, 1981, p. 50.

5. Cf. D. Séguillon, Une Histoire "à corps et à cri», catalogue de l'exposition, Institut National des Jeunes Sourds de Paris, 1994, p. 69 sqq.

6. Children of Silence est en fait le titre d'un livre de J.A. Seiss, Children of Silence: or a Story of the Deaf, Philadelphia, Porter and Coates, 1887.

Il n'est en outre pas sans intérêt, comme nous le verrons plus loin, que Dieu, même s'il n'est ici qu'un "lesser god ", un "sous-dieu " pourraiton dire, se trouve remplacé (traduit?) par l'idée du silence.

7. Un journal ne présente-t-il pas l'actrice sourde Emmanuelle Laborit comme la "porte-parole du monde du silence"!

8. Fantasme qui fonde sans doute également le soi-disant rapport du sourd à l'animalité, constamment présent lui aussi dans le discours entendant sur le sourd. Cf. M. Poizat, La Voix sourde, p. 48sqq. 9. Silence relatif d'ailleurs, car il accompagne souvent son énonciation en LSF d'émissions expressives vocales inarticulées, tout comme, de façon symétrique, l'entendant qui parle accompagne son énonciation de gestes expressifs mais "inarticulés", pourrait-on dire, car ne constituant pas un système de signifiants au sens strict.

10. Définition du "Petit Robert"

11. M. Poizat, La Voix sourde, déjà citée.

12. J. Derrida, La Voix et le Phénomène, Paris, P.U.F., 1967, p. 86.
13. Saint Augustin dans Les plus beaux sermons de saint Augustin, Paris, Études augustiniennes, tome III, 1986, p. 206-215.

14. J.C. Milner, L'Amour de la langue, Paris, Seuil, 1978, p. 8.

15. Fauchet (M. l'abbé), Oraison funèbre de Charles-Michel de l'Épée, Paris, J.R. Lottin, 1790, p. 32,34.

16. F. Berthier, Sur l'opinion de feu le Dr Itard relative aux facultés intellectuelles et aux qualités morales des Sourds-Muets. Réfutation présentée aux académies des Sciences morales et politiques, Paris, Michel Lévy frères, 1852 , p. 62.

17. Le lecteur intéressé pourra se reporter à nos développements de cette question dans La Voix sourde, déjà citée.

18. J. Lacan, Écrits, Paris, Seuil, 1966, p. 247 ; c'est nous qui soulignons. 19. C'est ce qui fait qu'on peut parler par exemple à son ordinateur - généralement pour l'insulter! (parce qu'il peut inscrire comme réponse sur l'écran un message - ou une absence de message), mais pas à la feuille de papier sur laquelle on écrit.

20. Bloch et von Wartburg, Dictionnaire étymologique, Paris, P.U.F.

21. C. Casagrande et S. Vecchio, Les Péchés de la langue, trad. de I Peccati della lingua par P. Baillet, Paris, Le Cerf, 1991, p. 313.

22. L.A. Walker, A Loss for Words, New York, Harper and Row, 1986, p. 36 .

23. Lacan repère en effet la structure psychotique de James Joyce qu'il analyse dans son séminaire "Le Sinthomme».

24. G. Büchner, Lenz (1879), trad. de l'allemand par B. Kreis, Nîmes, Jacqueline Chambon, 1991, p. 57.

25. Ibid.

26. V. Jankélévitch, La Musique et l'Ineffable, Paris, Seuil, 1983, p. 172.

27. Ibid., p. 173

28. Bien que toute la matière du troisième acte fût prête et qu'il manifestât à plusieurs reprises son désir de le terminer, Schönberg n'entreprit jamais la composition du troisième acte.

39. "Ma langue n’est pas déliée,

Je peux penser

mais non parler (discourir)".

30. « O Parole, Parole, toi qui me manques». 